\title{
The thermal history of crustal magmas: Why, How and Whaaaat?
}

\author{
ADAM J.R. KENT ${ }^{1} \&$ RICHARD W. BRADSHAW ${ }^{2}$
}

\author{
'Oregon State University, Corvallis OR, 97330, USA, \\ adam.kent@oregonstate.edu \\ ${ }^{2}$ Vanderbilt University, Nashville, Tennessee, USA.
}

There is broad consensus that the magmatic systems that underlie silciic volcanoes are transcrustal in vertcal extent. These systems are also likely to be highly variable in terms of the location and geometry of magma storage zones, and the compositions and relative abundances of crystal, liquid and vapour components. However, crustal magma systems will also evolve and reoragnize in response to changes in magma fluxes, crustal conditions and other external and internal forcing. Although a major research goal is to describe the state and distribution of different magmatic components, a full understanding of magmatic plumbing systems also requires an knowledge about temporal changes.

An important constraint on magmatic systems is the thermal conditions experienced by magmas through time, a.k.a. the thermal history, as this exerts a primary control on both the compositional and rheological state of magma. Furthermore, evidence suggests magmas are commonly resident in the crust for long periods - thousands to hundreds of thousands of years - and may experience a range of thermal conditions during this time. Although the thermal history of magmas has historically been difficult to measure, more recently a number of petrological and geochemical tools have been developed that constrain the thermal history of silicic magmas. These techmniques rely on different types of radiometric age dating, as well as quantifying thermal conditions via the observed extent of trace metal diffusion in minerals. To date, intepretation of these results has largely been characterized in terms of simplified "cold" vs. "warm" magma storage models. However reality is turning out to be far more interesting and variable, and suggests that constraining the thermal history of different magmas and magmatic components within and between different magmatic systems provides novel insight into the dynamics and evolution of different silicic magma systems.

Herein I will briefly review some of the techniques that provide insight into the thermal history of magmas, and discuss their strengths and limitations. New data suggests that we can discern differences in the thermal history of different magmatic components from the same system, and reinforces the value of this approach for deciphering the complexities of crustal magma storage through time in silicic magma systems. 